\title{
METHOD EXPERIMENTAL DEFINITION OF EFFICIENCY FUELBURN IN A GAS REACTORS WITH MINI CHANNELS
}

\author{
Ekaterina Slesareva ${ }^{1, *}$, Sergey Elistratov ${ }^{2}$, and Valeriy Ovchinnikov ${ }^{1}$ \\ ${ }^{1}$ SB RAS, Kutateladze Institute of Thermophysics, 630090 Novosibirsk, Russia \\ ${ }^{2}$ Novosibirsk State Technical University, 630092 Novosibirsk, Russia
}

\begin{abstract}
Approbation of a method of experimental definition of efficiency of a fuelburn in minichanneling gas reactors has been carried out. The method of visualisation of a field of temperatures by the temperature transducer made of fine-meshed with low-inertia use for definition of thermal structure of gas streams. A field of temperatures on an outlet the simulator of a gas reactor with minichannels registered on thermogram by infrared imager. Experiments have shown that the thermal imaging method provides the good resolutions on spatial and on time for a field of temperatures both in stationary and in non-stationary regime of gas flow and of heat release. Qualitative and quantitative character a change a temperatures on thermograms correspond to classical physical representations about nature of a gas flow in lengthy channels. The study showed that the method of thermal imaging allows in a regime real time defined a parameters of gas flow on outlet a channels of reactor which are necessary for definition of fuelburn efficiency.
\end{abstract}

\section{Introduction}

The mini-microchannel gas reactors and the heat-exchange apparatus become an integral part of innovative directions a machine industry of heat power and energy-chemical. Data about composition and temperature the gaseous reaction products, gained immediately on an outlet from such apparatuses, allow to optimise their operation.

The thermographic method an experimental study a definition of thermal structure a gas flow [1] is applied. For visualisation a pattern of temperature a medium which transparent in an infrared light, possible used thin filaments or fine-meshed heated up in a stream as a indicators of temperature. The thermographic method possible to trace changes both common and an local pattern a changing a temperature in a gas flow. In the present paper results of approbation the thermographic method [2] for heat exchangers with minichannel are presented.

\footnotetext{
* Corresponding author: styuardessa@yandex.ru
} 


\section{A measurement procedure}

Experiments on measuring non-stationary a field of temperature on an outlet from six longitudinal minichannels of quasitriangular cross-section have been carry out on the model framework organised by seven cylindrical heaters of equal diameter. On fig. 1 the crosssection and the passing channel in framework are schematically presented. In a quartz tube 1 outer diameter $23 \mathrm{~mm}$ and length $0.5 \mathrm{~m}$ disposes framework from seven electric heaters 2 diameter $5.9 \mathrm{~mm}$ and length $0.5 \mathrm{~m}$ which organising six passing minichannels 3 (№ $1 \div$ № 6) the quasitriangular shape (triangle $\mathrm{ABC}$ organised by archs $\mathrm{AB}, \mathrm{BC}, \mathrm{CA}$ ). Kaolinite wool 4 in thickness $7 \mathrm{~mm}$ and landlocked an air spaces 5 reduced heat loss from heaters in a circumambient.
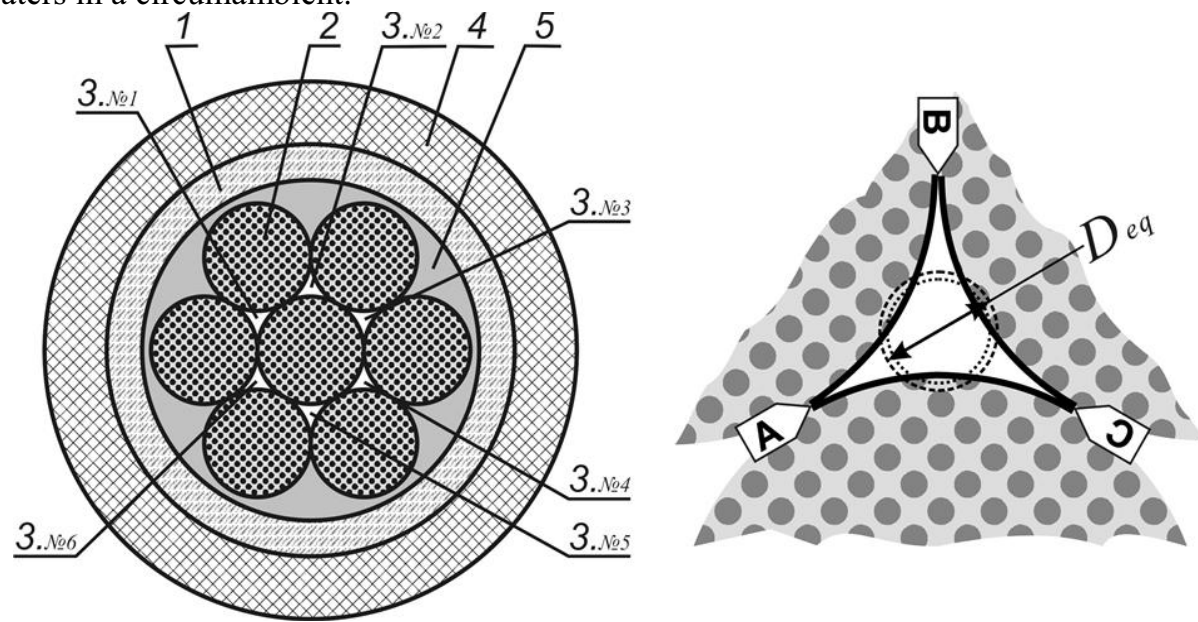

Fig. 1. A cross-section (a) and the shape (b) the passing quasitriangular channel of minichannel framework: 1 - a quartz tube; 2 - a heater; 3 - the passing channel (№ $1 \div$ № 6) for gas; 4 - thermo insulation; 5 - an air space; A, B and C - vertex of the quasitriangular channel.

The total heat exchange area passing quasitriangular channels is $0.0275 \mathrm{~m}^{2}$. On fig. $1 \mathrm{~b}$ the visual relationship a sizes the passing channel and its hydraulic diameter $D_{\text {eq }}$ is shown. By shaped lines rounds for minimum $(1.2 \mathrm{~mm})$ and maximum $(1.3 \mathrm{~mm})$ meanings of hydraulic diameters in framework are shown. The odds in diameters is caused by inaccuracy at mount a electric heaters in a bundle and some ovality of cylindrical heaters. According to planimetric measuring of photographs the sectional area the passing channels varied from 2.7 to $3.0 \mathrm{~mm}^{2}$. The brass mesh-thermode from a wire in the thickness about 80 microns with meshes $0.3 \times 0.3 \mathrm{~mm}$ press oneself to surface of channels on outlet framework. Thermogram have been obtained by infrared imager NEC TH7100 with wavelength thermal radiation $\lambda=8 \div 12$ microns.

\section{Discussion of results}

The thermographic method has been approved for various a flow rate of air and an intensity of its heating by cylindrical heaters in stationary and nonsteady conditions. Processing a thermograms has allowed determine a changes type a flow temperature in each a passing minichannels of framework. On fig. 2 four sample thermograms from the beginning (000) and after 99, 304, and 557 seconds after heat load $4.8 \mathrm{Wt}$ surge on a central heater are shown, under the total flow rate a air $0.45 \mathrm{~g} / \mathrm{s}$. It is possible to see inhomogeneity a warmup of framework because of a thermogravitational convection by volume assembly and 
variations a heating on the separate passing channels, caused by differences of their flow areas, noted previously.

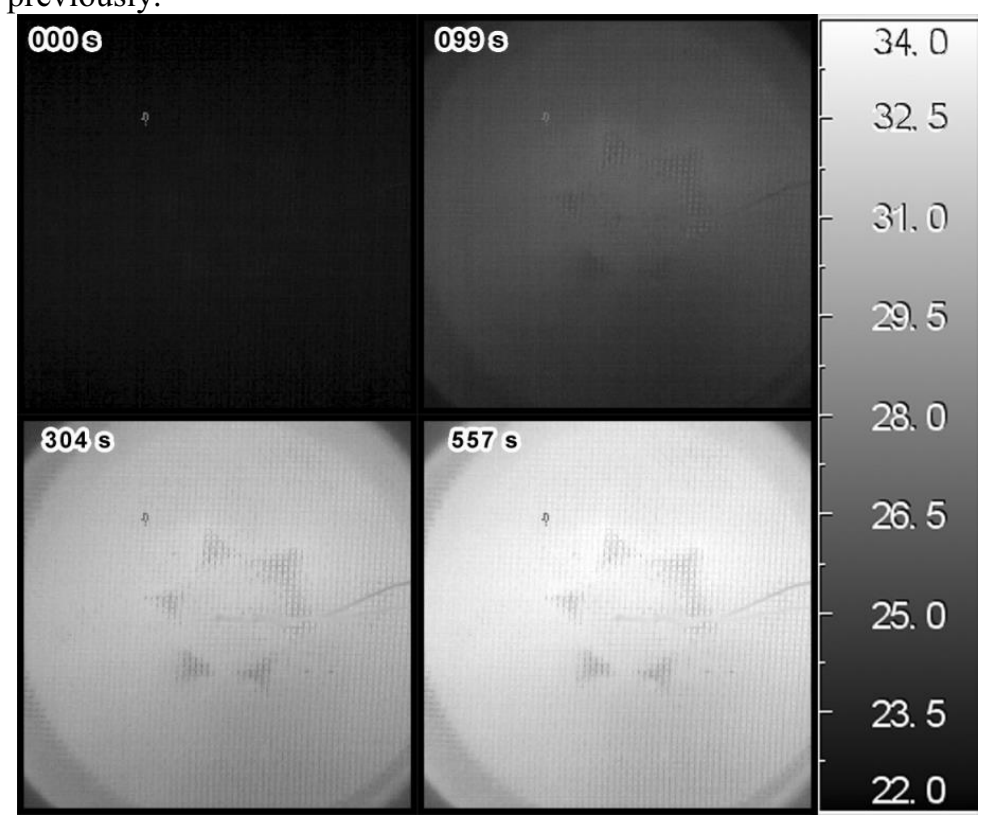

Fig. 2. The thermograms of mesh-thermode on outlet of a framework after heat load surge only on a central heater in a assembly.

Analogously on fig. 3 thermograms for case a decrease total flow rate air with $0.45 \mathrm{~g} / \mathrm{s}$ to $0.26 \mathrm{~g} / \mathrm{s}$ are shown, under a heat load $33.6 \mathrm{Wt}$ proportioned uniformly on all a heaters. The thermograms allow to see as all panoramic pattern of nonsteady preheating a air in quasitriangular minichannels, and full details a dynamic preheating of model framework.

Processing a thermograms has shown, that after transition to a stationary conditions the temperature of air on outlet a framework correspond to a calculated values for a stationary flow, taking into account changing a conditions of heat exchange along length of the channel $[3,4]$. Let's note, that for uprating accuracy of measuring by operation with channels of a smaller size it is necessary to use infrared imagers with smaller wavelength thermal radiation $\lambda$ for traceable caloradiance.

\section{Conclusion}

Approbation the thermographic method flow with using fine-meshed grids-thermode for definition a thermal structure a gas flow on outlet the simulation framework with minichannels has shown its good a spatial-time resolution. Qualitative and quantitative character a change a temperatures on thermograms correspond to classical physical representations about nature of a gas flow in lengthy channels [3]. 


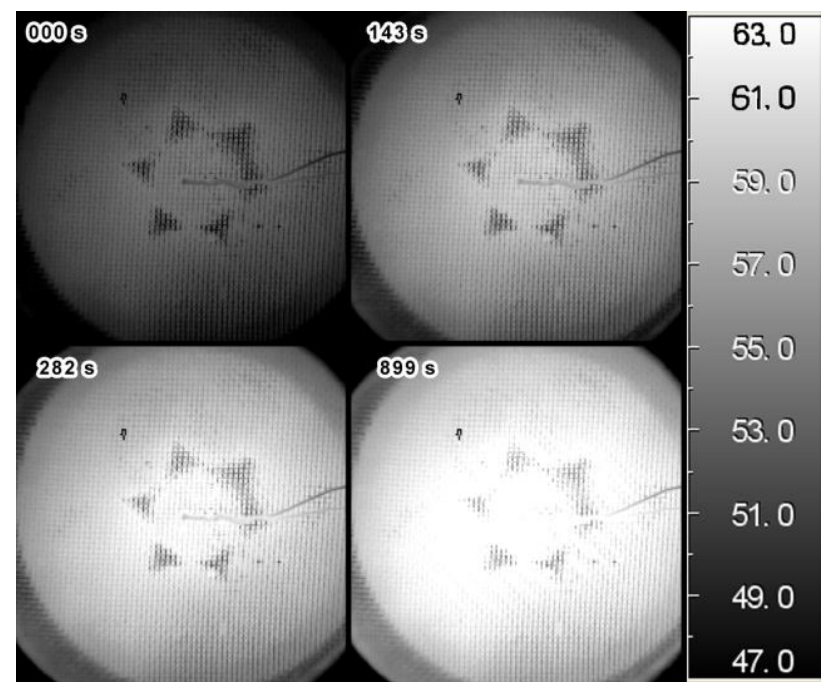

Fig. 3. The thermograms of mesh-thermode on outlet of a framework after a decrease total flow rate air.

The thermographic method is universal, its specific application for study a processes of stationary and nonsteady heat exchange, dependent from a specificity a subject of inquiry and engineering possibilities a equipment for thermal imaging.

\section{Acknowledgments}

This work was supported by the Russian Foundation for Basic Research, project No. 16-38-00502-mol-a.

\section{References}

1. A.V. Efimova, A.V. Zaitsev, B.P. Zhilkin, D.N. Tokarev, K.V. Zaitsev, Kh. Dashpuntsag, Bulletin of USTU: Therm. Eng. 33 (2004)

2. S.L. Elistratov, E.Yu. Slesareva, EPJ Web Conf. 82 (2015)

3. B.S. Petuhov, Heat exchange and resistance under a laminar flow fluid in tubes. (Energy, Moscow, 1967)

4. S.Y. Misyura, EPJ Web of Conf. 110 (2015) 